\title{
The Impact of Mild Cognitive Impairment on Gait and Balance: A Systematic Review and Meta-Analysis of Studies Using Instrumented Assessment
}

\author{
Lindsay Bahureksa $^{a}$ Bijan Najafi $^{a}$,,$f$ Ahlam Saleh ${ }^{c}$ Marwan Sabbagh ${ }^{d}$ \\ David Coon ${ }^{\mathrm{e}}$ M. Jane Mohler ${ }^{\mathrm{a}, \mathrm{b}}$ Michael Schwenk ${ }^{\mathrm{a}, \mathrm{b}, \mathrm{g}}$ \\ ${ }^{a}$ Interdisciplinary Consortium on Advanced Motion Performance (iCAMP), Department of Surgery, College of \\ Medicine, ${ }^{b}$ Arizona Center on Aging, and 'Arizona Health Sciences Library, University of Arizona, Tucson, Ariz., \\ ${ }^{d}$ Cleo Roberts Center for Clinical Research, Banner Sun Health Research Institute, Sun City, Ariz., e College of Nursing \\ and Health Innovation, Arizona State University, Phoenix, Ariz., and ${ }^{\mathrm{f}}$ Interdisciplinary Consortium on Advanced \\ Motion Performance (iCAMP), Michael E. DeBakey Department of Surgery, Baylor College of Medicine, \\ Houston, Tex., USA; ${ }^{9}$ Network Aging Research (NAR), Heidelberg University, Heidelberg, Germany
}

\section{Key Words}

Mild cognitive impairment - Gait · Balance - Technology ·

Older adults $\cdot$ Assessment $\cdot$ Analysis

\begin{abstract}
Background: In addition to cognitive deficits, people with mild cognitive impairment $(\mathrm{MCl})$ can experience motor dysfunction, including deficits in gait and balance. Objective, instrumented motor performance assessment may allow the detection of subtle $\mathrm{MCl}$-related motor deficits, allowing early diagnosis and intervention. Motor assessment under dualtask conditions may increase diagnostic accuracy; however, the sensitivity of different cognitive tasks is unclear. Objective: To systematically review the extant literature focusing on instrumented assessment of gait and balance parameters for discriminating $\mathrm{MCl}$ patients from cognitively intact peers. Methods: Database searches were conducted in PubMed, EMBASE, Cochrane Library, PsycINFO and Web of Science. Inclusion criteria were: (1) clinically confirmed $\mathrm{MCl}$; (2) instrumented measurement of gait and/or balance; (3) English lan-
\end{abstract}

\section{KARGER}

() 2016 S. Karger AG, Basel

E-Mail karger@karger.com

www.karger.com/ger guage, and (4) reporting gait or balance parameters which could be included in a meta-analysis for discriminating between $\mathrm{MCl}$ patients and cognitively intact individuals based on weighted effect size (d). Results: Fourteen studies met the inclusion criteria and reported quantitative gait $(n=11)$ or postural balance $(n=4)$ parameters to be included in the meta-analysis. The meta-analysis revealed that several gait parameters including velocity $(d=-0.74, p<0.01)$, stride length $(d=-0.65, p<0.01)$, and stride time (mean: $d=0.56$, $p=0.02$; coefficient of variation: $d=0.50, p<0.01$ ) discriminated best between $\mathrm{MCl}$ and healthy controls under singletask conditions. Importantly, dual-task assessment increased the discriminative power of gait variables wherein gait variables with counting tasks appeared to be more sensitive (range $d=0.84-1.35$ ) compared to verbal fluency tasks such as animal naming (range $d=0.65-0.94$ ). Balance parameters identified as significant discriminators were anterior-posterior $(\mathrm{d}=0.49, \mathrm{p}<0.01)$ and mediolateral $(\mathrm{d}=-0.34, \mathrm{p}=0.04)$ sway position in the eyes-open condition but not eyesclosed condition. Conclusion: Existing studies provide evidence that $\mathrm{MCl}$ affects specific gait parameters. $\mathrm{MCl}$-related 
gait changes were most pronounced when subjects are challenged cognitively (i.e., dual task), suggesting that gait assessment with an additional cognitive task is useful for diagnosis and outcome analysis in the target population. Static balance seems to also be affected by $\mathrm{MCl}$, although limited evidence exists. Instrumented motor assessment could provide a critical opportunity for $\mathrm{MCl}$ diagnosis and tailored intervention targeting specific deficits and potentially slowing progression to dementia. Further studies are required to confirm our findings.

(C) 2016 S. Karger AG, Basel

\section{Introduction}

Along with research on dementia, there is an increased interest in mild cognitive impairment (MCI), a transitional cognitive state with a $10-15 \%$ yearly progression to dementia [1]. The precise diagnosis of MCI may allow early intervention and prevention of a further cognitive and functional decline [2]. To date, $16 \%$ of individuals above the age of 70 years have been diagnosed with MCI [3]. By 2050, it is estimated that 1 in 85 persons will be diagnosed with Alzheimer's disease [4], and MCI has become the focus of studies for early diagnosis and potential intervention.

MCI is characterized by: (1) preserved general cognitive function, (2) objective memory impairment beyond age, (3) lack of dementia, and (4) little or no impairment of activities of daily living (ADL) [5-7]. Despite relatively preserved ADL function, studies have reported subtle changes in functional performances such as gait and balance in people with MCI. Although these changes do not cause a drastic decline in everyday function [8], they may be clinically relevant and lead to motor errors in mobility task falls. Thus, early identification of subtle MCI-related changes in gait and balance might be relevant for targeting specific interventions aiming to prevent further decline $[9,10]$. Conventional gait and balance tests may, however, not be sufficiently accurate for the detection of subtle MCI-associated motor impairments [11]. Recent advances in electronic gait analysis and wearable technology may allow a more precise estimation of MCI-related changes in motor performance. Many spatiotemporal gait variables can be extracted and several seem to be associated with cognitive decline [12]. Identification of gait parameters which are strongly associated with MCI could be relevant for early diagnosis and intervention. However, to our knowledge, a systematic review and meta-analysis comparing instrumented gait variables in people with MCI and healthy controls has not been performed.
Further, dual-task gait assessment may be more helpful to detect cognition-related gait changes as compared to single-task assessment [10,13]. Similarly, to our knowledge, it has not been systematically investigated whether a gait assessment under dual-task conditions has an added value in detecting gait dysfunction in MCI patients.

As opposed to dynamic balance assessed during walking, static postural balance during standing is another motor function that is critical to quality of life and seems to have a direct association with cognitive function [14]. However, it has not been systematically investigated which specific balance parameters derived from an instrumented static balance assessment (e.g., posturography) are linked to MCI.

Our objective was to systematically review the extant literature focusing on instrumented assessment of gait and balance parameters for discriminating clinically confirmed MCI patients from cognitively intact older adults.

\section{Methods}

This review was performed to be consistent with the PRISMA statement [15]. Searches were conducted in July 2015 in the following databases: PubMed (1946-2015); Thomson Reuters Web of Science (Science Citation Index Expanded 1900-2015; Conference Proceedings Citation Index Science 1990-2015); Wiley Online Library Cochrane Library (1898-2014); EBSCO PsycINFO (1597-present), and Embase.com EMBASE (1947-2015). The search strategy for PubMed can be found in online supplementary appendix A (see www.karger.com/doi/10.1159/000445831 for all online suppl. material) and was adapted for all other databases. The reference lists of related reviews on cognition, balance, and gait were also searched for eligible papers.

Inclusion criteria consisted of: (a) population: individuals with confirmed MCI diagnosis according to established definitions (e.g., Petersen [5], Winblad et al. [16]); (b) type of outcome measures: gait variables obtained by instrumented analysis (e.g., electronic walkways, wearable sensors, and camera systems) or static postural balance variables obtained by instrumented analysis (e.g., stabilometry); (c) original article, and (d) English language. Articles that only used a stopwatch were excluded, as were articles that did not provide data which could be utilized in meta-analysis (i.e., mean and standard deviation) or which included a population with comorbid gait disorders (e.g., Parkinson's disease).

Two reviewers (L.B. and Tulcy Patel) independently screened the titles and abstracts from the initial search to identify potentially relevant records. If the reviewers were unable to determine a study's eligibility based on the title and abstract, the full text was retrieved. A third reviewer (M.S.) resolved disagreements between the two screenings. Selected full texts were then reviewed for inclusion, per PRISMA protocol.

Data extraction of the study characteristics and findings was performed by a single reviewer (L.B.). Study characteristics of interest were: (1) main goal of the study; (2) type of MCI definition; (3) participant characteristics, and (4) key results of the study with
68

Gerontology 2017;63:67-83 DOI: $10.1159 / 000445831$
Bahureksa/Najafi/Saleh/Sabbagh/Coon/ Mohler/Schwenk 
Fig. 1. Flowchart of the process of initial literature search and extraction of studies meeting the inclusion criteria.

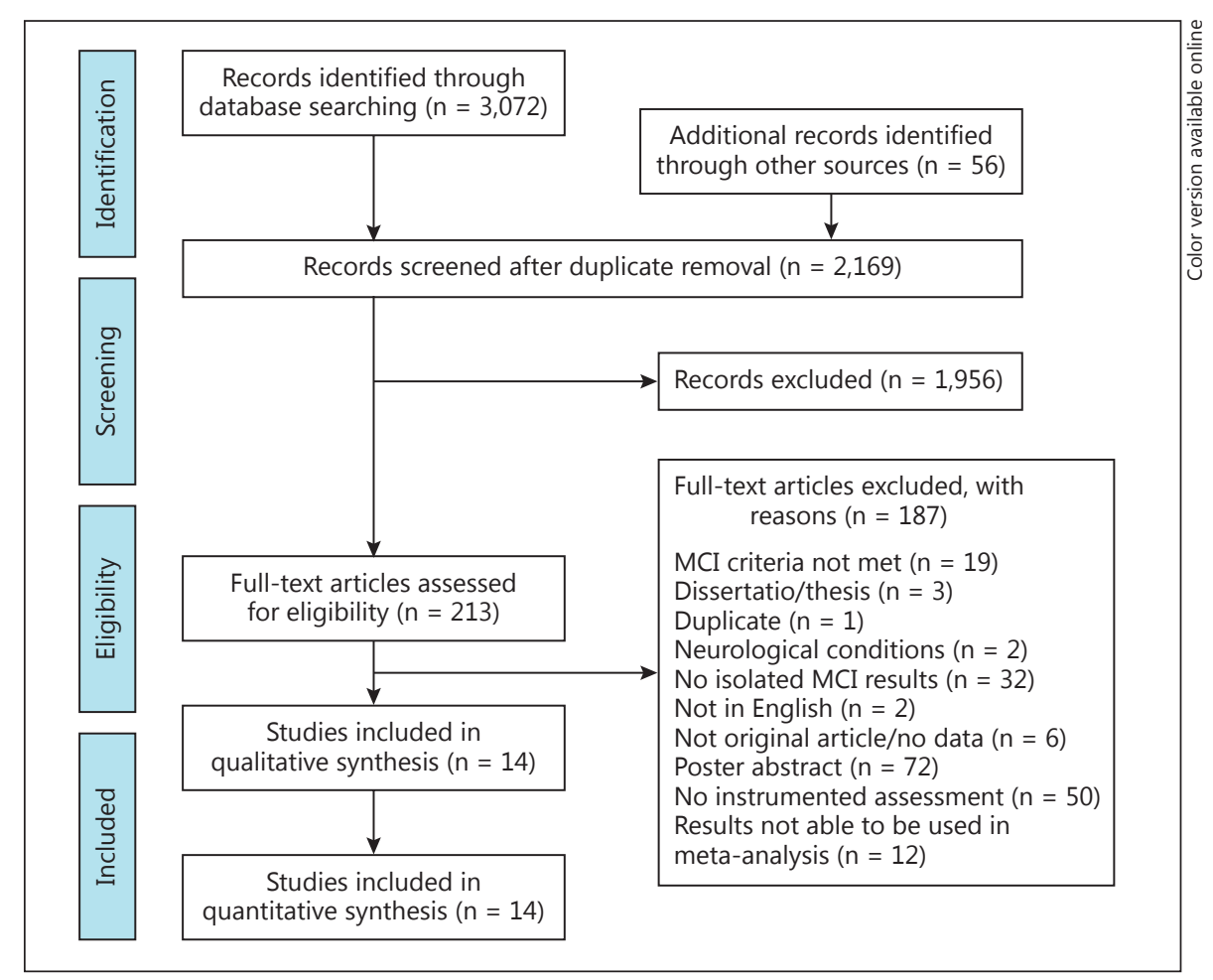

respect to gait and balance. In 2 papers $[17,18]$ where the $p$ value was not reported but the sample size was sufficient to be approximated as normal distributions, the $\mathrm{p}$ value was calculated using independent $t$ tests between the cognitively healthy and MCI groups. Assessment of the methodological quality of each study was performed using the Cochrane Collaboration tool for assessing the risk of bias (online suppl. appendix B).

\section{Meta-Analysis}

In order to estimate the discriminative power (i.e., MCI vs. healthy control) of specific gait and balance variables, a meta-analysis was conducted for each variable reported in two or more studies. The outcome of each meta-analysis was the overall effect size (Cohens' d), representing the standardized mean difference between a study group of cognitively healthy individuals (CHI) and a study group with individuals with MCI. The Cohen criteria were used for interpretation (small: $\mathrm{d}>0.2$, medium: $\mathrm{d}>0.5$, large effect: $\mathrm{d}>0.8$ ) [19].

Positive effect sizes were indicative of an increase in the gait/ balance parameter value in subjects with MCI when compared to CHI. Likewise, negative effect sizes indicated a decrease in the gait/ balance parameter value. Heterogeneity was assessed using Cochran's Q and $\mathrm{I}^{2}$. When studies were homogeneous (Cochran's Q $<0.05, \mathrm{I}^{2}>0.75$ ), the effect sizes were calculated using inverse variance analysis; when studies were heterogeneous, the effect sizes were calculated using random effects analysis. The mean effect sizes, $95 \%$ confidence intervals (CI), Cochran's Q, and $\mathrm{I}^{2}$ were calculated for each parameter and used to create forest plots for visualization of the meta-analysis using the MetaXL software (version 2.2, EpiGear, Wilston, Qld., Australia). Assessment of publication bias was performed by generating a funnel plot for the most fre-

Impact of MCI on Balance and Gait quently reported gait variable (i.e., single-task gait velocity) (online suppl. appendix C). Other gait/balance parameters were reported only in a limited number of studies; therefore, assessment of publication bias via funnel plots was not possible.

\section{Results}

The database searches yielded 3,072 papers, with an additional 56 papers found through searching reference lists. After removal of duplicates and title/abstract screening, 213 papers remained for full-text screening. Of these, 14 met the inclusion criteria (fig. 1). The majority of the studies $(n=11,78.6 \%$ ) focused on the interaction of MCI and gait, while a smaller percentage $(n=4,28.6 \%)$ focused on balance. One study included both gait and balance analysis [11]. Motor parameters were obtained by wearable sensors, force plates, and electronic walkways such as the GAITRite (table 1).

The most frequently used definitions of MCI were those by Winblad et al. [16] $(n=6)$ and Petersen [5] $(n=$ 4 ), and some papers using these criteria additionally identified amnestic or nonamnestic MCI subtypes (a-MCI, na-MCI) $(n=3)$. Miscellaneous cognitive criteria $(n=3)$ that adhered to the MCI standard were also included in the analysis (table 2). 
Table 1. Instruments used in the assessment of balance and gait

\begin{tabular}{lll}
\hline Instrument & Papers & \\
\cline { 2 - 3 } & $\mathrm{n}(\%)$ & citations \\
\hline Electronic walkways & $8(57.1)$ & {$[17,18,20-22,24-26]$} \\
Body-worn sensors & $3(21.4)$ & {$[11,23,27]$} \\
Force plates & $3(21.4)$ & {$[29-31]$} \\
\hline
\end{tabular}

\section{Gait Parameters Reported in Studies}

Participants

Of the 11 studies that focused on gait, 10 studies compared MCI subjects with healthy age-matched controls $[11,17,18,20-26]$. Five papers $[11,18,20,23,26]$ additionally examined differences between individuals with MCI and dementia. In 3 papers [21, 24, 25], subtype differences between a-MCI and na-MCI were additionally examined (table 3 ).

\section{Parameters}

Gait parameters and assessments varied substantially amongst the studies, even when the same instrument was used for evaluation. A summary of the studies that used gait assessment is presented in table 3. Eleven papers reported quantitative gait data, which included gait velocity $(n=10)$, gait velocity variability $(n=6)$, stride time variability $(n=6)$, stride time $(n=4)$, stride length $(n=2)$, stride frequency $(n=1)$, swing time $(n=1)$, and step regularity $(n=1)$. This paper focuses on the parameters that were reported in 2 or more papers (e.g., gait velocity, stride length, stride time, and stride time variability). Qualitative results are provided for single papers that could not be included in meta-analysis.

\section{Effect of MCI on Gait Parameters}

Gait Velocity

Among articles which reported single-task gait velocity, 5 studies [17, 22, 24-26] found a significant decrease in subjects with MCI in comparison to those who are cognitively healthy, whereas $5[11,18,20,21,27]$ did not identify a significant difference. Pooling of data within a meta-analysis of 10 eligible studies showed a moderate to large significant effect ( $\mathrm{d}=-0.74,95 \% \mathrm{CI},-0.89$ to -0.59 , $\mathrm{p}<0.001$; fig. 2).

Dual-task conditions were examined in 5 papers. Dual-task gait velocity was significantly slower in persons with MCI during backwards counting by 7's $(\mathrm{n}=3)[17$, $18,28]$, backwards counting by 1 's $(\mathrm{n}=2)[11,26]$ and
Table 2. Criteria for MCI reported in studies

\begin{tabular}{lll}
\hline Criteria & Papers & \\
\cline { 2 - 3 } & $\mathrm{n}(\%)$ & citations \\
\hline Petersen et al. [5, 59] & $4(28.5)$ & {$[22,24,25,30]$} \\
Winblad et al. [16] & $6(42.8)$ & {$[17,18,20,21,26,31]$} \\
CERAD & $1(7.1)$ & {$[23]$} \\
Miscellaneous & $3(21.42)$ & {$[11,27,29]$} \\
\hline
\end{tabular}

CERAD = Consortium to Establish a Registry for Alzheimer's Disease.

animal naming $(\mathrm{n}=3)[17,18,26]$ in comparison to cognitively intact peers. Meta-analysis of these papers revealed significant differences between MCI and healthy controls in all three conditions, with the largest effect found for counting backwards by 7's $(\mathrm{d}=-1.34,95 \% \mathrm{CI}$, -1.74 to -0.93 , $\mathrm{p}<0.01$; fig. $3 \mathrm{a}$ ) and with counting backwards by 1 's ( $\mathrm{d}=-0.92,95 \% \mathrm{CI},-1.19$ to $-0.66, \mathrm{p}<0.01$; fig. $3 \mathrm{~b})$ and animal naming $(\mathrm{d}=-0.94,95 \% \mathrm{CI},-1.20$ to $-0.68, \mathrm{p}<0.01$; fig. $3 \mathrm{c}$ ) having similar effect sizes.

\section{Stride Length}

Stride length was examined in 3 studies $[11,24,28]$ for single-task, and in 2 studies $[11,28]$ for dual-task conditions. In single-task conditions, 1 paper [24] identified a significant decrease in stride length for both a-MCI and na-MCI subtypes in comparison to healthy controls, while 2 papers $[11,28]$ identified no significant effect of MCI. Meta-analysis was performed for single-task stride length for 2 papers $[11,24]$ where mean and standard deviation data was provided and revealed a significant medium effect ( $\mathrm{d}=-0.65,95 \% \mathrm{CI},-0.88$ to $-0.41, \mathrm{p}<0.01$; fig. 4a). Change in dual-task stride length was reported to be insignificant in 2 papers $[11,28]$, which were excluded from the meta-analysis since the studies used the same data set.

\section{Stride Time}

Under single-task conditions, the increase in stride time was significant in 2 studies [17, 23], and nonsignificant in another $2[18,27]$. The meta-analysis revealed that single-task stride time significantly discriminated between both groups with a medium effect size $(\mathrm{d}=0.56$, $95 \%$ CI, 0.23-0.89, $\mathrm{p}=0.02$; fig. $4 \mathrm{~b}$ ).

Under backwards counting (by 7's) and animal naming dual task, 2 studies $[17,18]$ reported significant differences in stride time between the MCI and CHI groups. 
Table 3. Summary of included studies involving gait and MCI

\begin{tabular}{|c|c|c|c|c|}
\hline $\begin{array}{l}\text { First author [Ref.], } \\
\text { year }\end{array}$ & Study characteristics ${ }^{1}$ & Instrumented assessment & Instrument & $\begin{array}{l}\text { Significant gait results in } \\
\text { MCI group }\end{array}$ \\
\hline $\begin{array}{l}\text { Beauchet [21], } \\
2011\end{array}$ & $\begin{array}{l}\text { Criteria: Winblad et al. }[16] \\
\text { CHI: } \mathrm{n}=21,70.3 \text { years } \\
\text { a-MCI: } \mathrm{n}=15,73.3 \text { years, } 42.9 \% \\
\text { na-MCI: } \mathrm{n}=21,70.6 \text { years, } 26.7 \%\end{array}$ & Walking at usual pace & $\begin{array}{l}\text { GAITRite Gold Walkway } \\
\text { (length: } 9.72 \mathrm{~m} \text { ) }\end{array}$ & $\begin{array}{l}\uparrow \text { Gait velocity variability } \\
\text { in a-MCI } \\
\text { No change in gait velocity } \\
\text { variability for na-MCI }\end{array}$ \\
\hline $\begin{array}{l}\text { Beauchet [20], } \\
2013\end{array}$ & $\begin{array}{l}\text { Criteria: Winblad et al. }[16] \\
\text { CHI: } \mathrm{n}=44,74.5 \text { years, } 63.6 \% \\
\text { MCI: } \mathrm{n}=39,73.6 \text { years, } 38.5 \% \\
\text { AD: } \mathrm{n}=33,79.2 \text { years, } 63.6 \%\end{array}$ & $\begin{array}{l}\text { Walking at usual pace } \\
\text { Walking at fast pace }\end{array}$ & $\begin{array}{l}\text { GAITRite Gold Walkway } \\
\text { (length: } 9.72 \mathrm{~m} \text { ) }\end{array}$ & $\begin{array}{l}\text { No change in STV at } \\
\text { normal walking velocity } \\
\uparrow \text { STV at fast walking } \\
\text { velocity }\end{array}$ \\
\hline $\begin{array}{l}\text { Boripuntakul [22], } \\
2014\end{array}$ & $\begin{array}{l}\text { Criteria: (a) Petersen et al. [5], } \\
\text { (b) MMSE } \geq 24,(\mathrm{c}) \text { MoCA }<26 \\
\text { CHI: } \mathrm{n}=30,71.0 \text { years, } 66.7 \% \\
\text { MCI: } \mathrm{n}=30,70.6 \text { years, } 66.7 \%\end{array}$ & $\begin{array}{l}\text { Gait initiation and walking } \\
\text { at usual pace } \\
\text { Gait initiation and walking } \\
\text { during counting dual task } \\
\text { (backwards by 7's) }\end{array}$ & $\begin{array}{l}\text { GAITRite system (length } \\
\text { not reported) }\end{array}$ & $\begin{array}{l}\uparrow \text { Swing time of 1st/ } \\
2 \text { nd step, both tasks } \\
\uparrow \text { Step length variability } \\
\text { of } 1 \text { st/2nd step, both tasks }\end{array}$ \\
\hline $\begin{array}{l}\text { Choi [23], } \\
2011\end{array}$ & $\begin{array}{l}\text { Criteria: CERAD-Korea } \\
\text { CHI: } n=6,71.6 \text { years, } 33.3 \% \\
\text { MCI: } n=7,72.9 \text { years, } 42.9 \% \\
\text { AD: } n=10,77.2 \text { years, } 60 \%\end{array}$ & $\begin{array}{l}\text { Walking at usual pace } \\
(25 \mathrm{~m})\end{array}$ & $\begin{array}{l}\text { Tri-axial accelerometer, } \\
\text { right foot }\end{array}$ & $\uparrow$ Stride time \\
\hline $\begin{array}{l}\text { Gillain [11], } \\
2007\end{array}$ & $\begin{array}{l}\text { Criteria: (a) cognitive disorder with } \\
\text { no major impact on ADL, (b) CDR } \\
<0.5 \text {, (c) MMSE } \geq 24 \\
\text { CHI: } \mathrm{n}=14,73.5 \text { years, } 21 \% \\
\text { MCI: } \mathrm{n}=14,72.9 \text { years, } 21 \% \\
\text { DEM: } \mathrm{n}=6,73.7 \text { years, } 9 \%\end{array}$ & $\begin{array}{l}\text { Single-leg balance test } \\
\text { Single-leg balance test with } \\
\text { dual task (countdown from } \\
50 \text { ) } \\
\text { Pull test } \\
\text { TUG test } \\
\text { TUG test with dual task } \\
\text { (countdown from 50) }\end{array}$ & $\begin{array}{l}\text { Locometrix }^{\circledR} \text { tri-axial } \\
\text { accelerometers }\end{array}$ & $\begin{array}{l}\text { Single tasking: } \downarrow \text { gait } \\
\text { symmetry } \\
\text { Dual tasking: } \downarrow \text { stride } \\
\text { frequency, gait velocity } \\
\text { positively correlates with } \\
\text { MMSE score }\end{array}$ \\
\hline $\begin{array}{l}\text { Montero-Odasso } \\
{[17], 2012}\end{array}$ & $\begin{array}{l}\text { Criteria: Winblad et al. }[16] \\
\text { CHI: } n=25,71.5 \text { years, } 88 \% \\
\text { MCI: } n=43,75.1 \text { years, } 54 \%\end{array}$ & $\begin{array}{l}\text { Walking at usual speed } \\
\text { Walking with dual task } \\
\text { (counting backward from } \\
100 \text { by } 7 \text { ) } \\
\text { Walking with dual task } \\
\text { (naming animals) }\end{array}$ & $\begin{array}{l}\text { GAITRite System (length: } \\
6 \mathrm{~m})\end{array}$ & $\begin{array}{l}\text { All assessments: } \\
\downarrow \text { gait velocity, } \uparrow \text { gait } \\
\text { variability, } \uparrow \text { stride time }\end{array}$ \\
\hline $\begin{array}{l}\text { Montero-Odasso } \\
{[25], 2014}\end{array}$ & $\begin{array}{l}\text { Criteria: Petersen }[5] \\
\text { a-MCI: } \mathrm{n}=42,77.3 \text { years, } 42 \% \\
\text { na-MCI: } \mathrm{n}=22,74.2 \text { years, } 64 \% \\
\text { CHI: } \mathrm{n}=35,70.4 \text { years, } 83 \%\end{array}$ & $\begin{array}{l}\text { Walking at usual speed } \\
\text { Walking with dual task } \\
\text { (counting backward from } \\
100 \text { by } 1 \text { ) } \\
\text { Walking with dual task } \\
\text { (counting backward from } \\
100 \text { by } 7 \text { ) } \\
\text { Walking with dual task } \\
\text { (naming animals) }\end{array}$ & $\begin{array}{l}\text { GAITRite System (length: } \\
6 \mathrm{~m})\end{array}$ & $\downarrow$ Gait velocity \\
\hline $\begin{array}{l}\text { Muir [18], } \\
2012\end{array}$ & $\begin{array}{l}\text { Criteria: Winblad et al. }[16] \\
\text { CHI: } n=22,71.0 \text { years, } 88 \% \\
\text { MCI: } n=29,73.6 \text { years, } 59 \% \\
\text { DEM: } n=23,77.5 \text { years, } 61 \%\end{array}$ & $\begin{array}{l}\text { Walking at usual speed } \\
\text { Walking with dual task } \\
\text { (counting backward from } \\
100 \text { by } 1 \text { ) } \\
\text { Walking with dual task } \\
\text { (counting backward from } \\
100 \text { by } 7 \text { ) } \\
\text { Walking with dual task } \\
\text { (naming animals) }\end{array}$ & $\begin{array}{l}\text { GAITRite System (length: } \\
6 \mathrm{~m})\end{array}$ & $\begin{array}{l}\text { All dual tasking: } \\
\downarrow \text { gait velocity, } \\
\uparrow \text { stride time, } \uparrow \text { STV }\end{array}$ \\
\hline $\begin{array}{l}\text { Nascimbeni [27], } \\
2015\end{array}$ & $\begin{array}{l}\text { Criteria: (a) MMSE, (b) digit span/ } \\
\text { Corsi span test, (c) short story } \\
\text { recall, (d) attention and visual } \\
\text { search } \\
\text { CHI: } \mathrm{n}=10,72.0 \text { years, } 40 \% \\
\text { MCI: } \mathrm{n}=13,76.0 \text { years, } 15 \%\end{array}$ & $\begin{array}{l}\text { Walking at usual speed } \\
\text { Walking with dual task } \\
\text { (phonemic fluency) } \\
\text { Walking with dual task } \\
\text { (short story recall) } \\
\text { Walking with dual task } \\
\text { (counting backward by 1's) }\end{array}$ & $\begin{array}{l}\text { Gait laboratory (length: } \\
12 \mathrm{~m} \text { ), STEP } 32 \text { Gait } \\
\text { analysis system }\end{array}$ & $\begin{array}{l}\text { Phonemic fluency dual } \\
\text { task: } \uparrow \text { double support } \\
\text { time, } \downarrow \text { gait velocity } \\
\text { Counting backwards dual } \\
\text { task: } \uparrow \text { double support } \\
\text { time }\end{array}$ \\
\hline
\end{tabular}


Table 3 (continued)

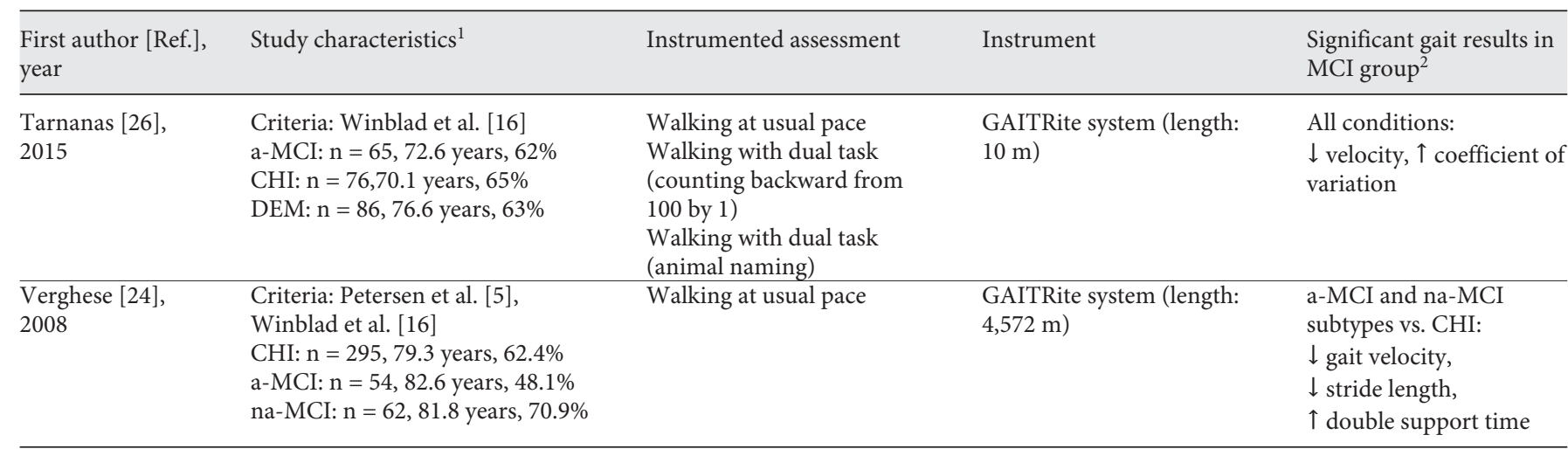

$\uparrow=$ Increased; $\downarrow$ = decreased; CDR = Clinical Dementia Rating; DEM = dementia including Alzheimer's disease; STV = stride time variability; TUG = timed up and go.

${ }^{1}$ Number, mean age, $\%$ female.

${ }^{2}$ Compared to an age-matched cognitively healthy control group, if present in the study.

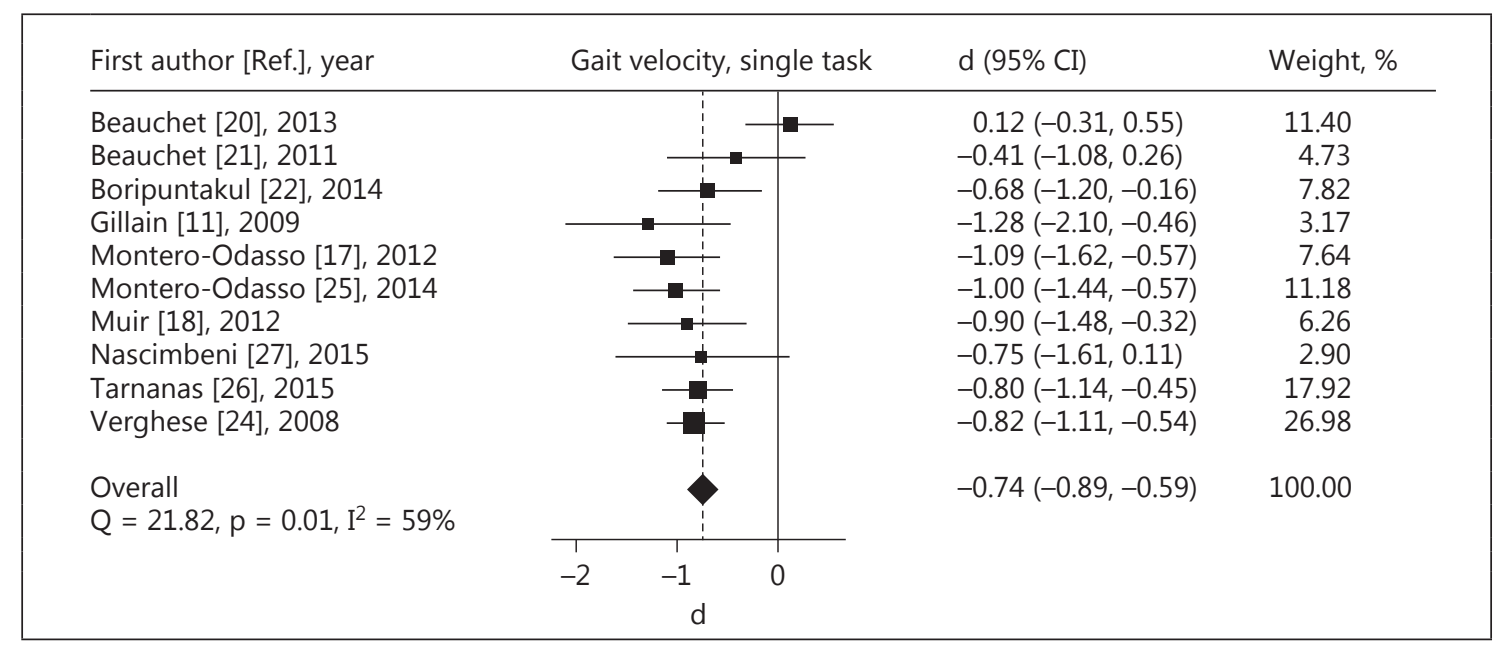

Fig. 2. Forest plot illustrating the effect of MCI on single-task gait velocity when compared to cognitively healthy controls. The dotted vertical line corresponds to the overall effect size, while the solid vertical line corresponds to no effect.

The meta-analysis revealed significant differences with a larger effect size for backwards counting by 7's $(\mathrm{d}=0.91$, 95\% CI, 0.53-1.30, $\mathrm{p}<0.01$; fig. 4c) compared to animal naming dual tasks ( $\mathrm{d}=0.84,95 \% \mathrm{CI}, 0.46-1.23, \mathrm{p}<0.01$; fig. $4 \mathrm{~d})$.

\section{Coefficient of Variation of Stride Time}

In 4 papers $[17,18,23,26]$, the increase in single-task stride time coefficient of variation $(\mathrm{CoV})$ was significant, while 2 papers [20,27] did not find that differences were significant. Analysis of these 6 papers revealed a medium positive effect that was significant $(\mathrm{d}=0.50,95 \% \mathrm{CI}, 0.29$ $0.71, \mathrm{p}<0.01$; fig. 5a).

For dual-task stride time CoV, Montero-Odasso et al. [17] found a significant increase during both backwards counting by 7's and animal naming, and 2 papers $[18,26]$ additionally found a significant increase in backwards counting by 1's dual-task conditions. The meta-analysis revealed a significant increase of dual-task stride time variability in MCI versus healthy subjects with larger effects for backwards counting tasks ( 1 's, $d=0.86,95 \% \mathrm{CI}$, $0.58-1.14$, $\mathrm{p}<0.01$, fig. 5b; 7's, $d=0.84,95 \%$ CI, $0.45-$ 


\begin{tabular}{|c|c|c|c|}
\hline First author [Ref.], year & Gait velocity, counting dual task (7's) & $d(95 \%$ CI $)$ & Weight, \% \\
\hline $\begin{array}{l}\text { Montero-Odasso [17], } 2012 \\
\text { Muir [18], } 2012\end{array}$ & & $\begin{array}{l}-1.21(-1.75,-0.68) \\
-1.51(-2.14,-0.88)\end{array}$ & $\begin{array}{l}58.09 \\
41.91\end{array}$ \\
\hline $\begin{array}{l}\text { Overall } \\
Q=0.49, p=0.48, I^{2}=0 \%\end{array}$ & & $-1.34(-1.74,-0.93)$ & 100.00 \\
\hline & $\mathrm{d}^{-1}$ & & \\
\hline
\end{tabular}

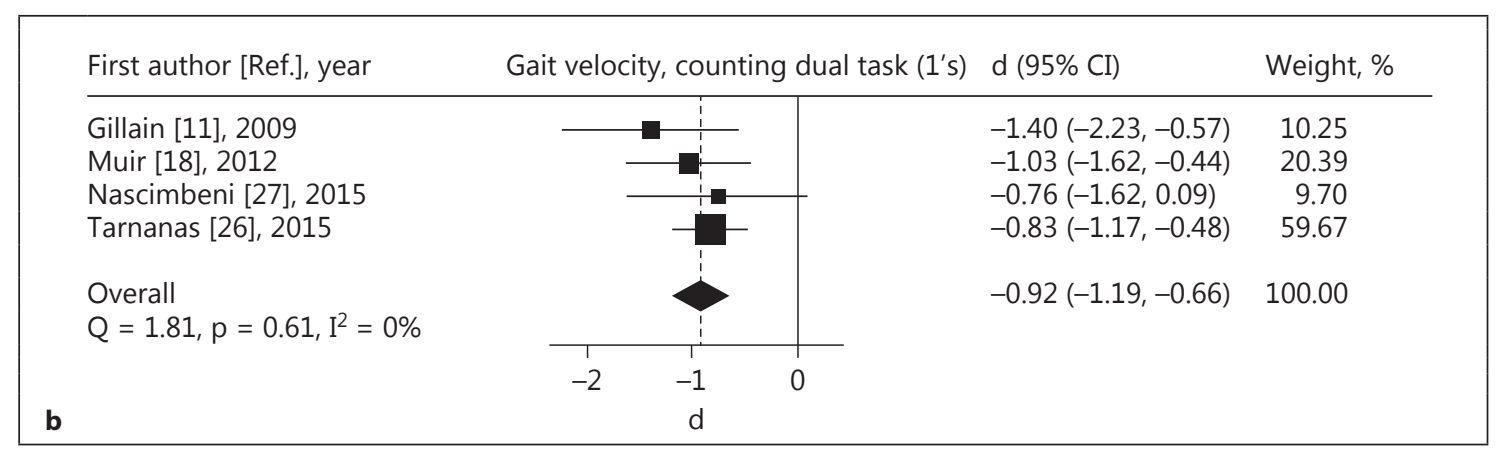

\begin{tabular}{|c|c|c|c|}
\hline First author [Ref.], year & Gait velocity, animal naming dual task & $d(95 \%$ CI $)$ & Weight, \% \\
\hline $\begin{array}{l}\text { Montero-Odasso [17], } 2012 \\
\text { Muir [18], 2012 } \\
\text { Tarnanas [26], } 2015\end{array}$ & $\begin{array}{l:l} & \\
& \\
& \\
\end{array}$ & $\begin{array}{l}-0.99(-1.52,-0.47) \\
-1.22(-1.82,-0.61) \\
-0.83(-1.17,-0.48)\end{array}$ & $\begin{array}{l}24.84 \\
18.49 \\
56.67\end{array}$ \\
\hline $\begin{array}{l}\text { Overall } \\
Q=1.25, p=0.54, I^{2}=0 \%\end{array}$ & & $-0.94(-1.20,-0.68)$ & 100.00 \\
\hline & $\begin{array}{l}-1 \\
d\end{array}$ & & \\
\hline
\end{tabular}

Fig. 3. Forest plot illustrating the effect of MCI on dual-task gait velocity during backwards counting by 7's (a), backwards counting by 1's (b), and animal naming (c) when compared to cognitively healthy controls. The dotted vertical line corresponds to the overall effect size.

$1.22, \mathrm{p}<0.01$, fig. $5 \mathrm{c})$, compared to animal naming $(\mathrm{d}=$ $0.51,95 \%$ CI, $0.26-0.76, \mathrm{p}<0.01$, fig. $5 \mathrm{~d}$ ).

\section{Qualitative Result}

One paper [22] specifically analyzed gait initiation using the GAITRite system. The authors reported a significantly increased step length and step width variability related to the walking condition (i.e., single vs. dual task) during gait initiation. Although mean spatiotemporal parameters (i.e., swing time, step time, step length, and step width) were not significantly different among the first two steps, variability in these parameters was reported to be significant between groups in all but one parameter (step time).
One study examined the effect of walking speed (i.e., habitual vs. fast walking) on outcomes [20]. Authors reported that MCI patients display a high stride time variability during fast-pace walking speed which was not seen at slower paces, and thus could be used as a specific biomarker of MCI patients.

\section{Balance Parameters Reported in Studies}

\section{Participants}

Four papers [11,29-31] focused on the interaction of MCI and balance. All 4 compared MCI subjects to cognitively healthy controls as well as subjects with mild-tomoderate dementia or dementia (table 4). 


\begin{tabular}{|c|c|c|c|}
\hline First author [Ref.], year & Single task stride length & $d(95 \%$ CI) & Weight, \% \\
\hline $\begin{array}{l}\text { Gillain [11], 2009 } \\
\text { Verghese [24], } 2008\end{array}$ & 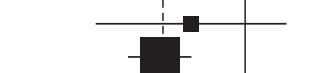 & $\begin{array}{l}-0.43(-1.18,0.32) \\
-0.67(-0.92,-0.42)\end{array}$ & $\begin{array}{r}9.97 \\
90.03\end{array}$ \\
\hline \multirow[t]{2}{*}{$\begin{array}{l}\text { Overall } \\
Q=0.35, p=0.56, I^{2}=0 \%\end{array}$} & & $-0.65(-0.88,-0.41)$ & 100.00 \\
\hline & -1 & & \\
\hline & $\mathrm{d}$ & & \\
\hline
\end{tabular}

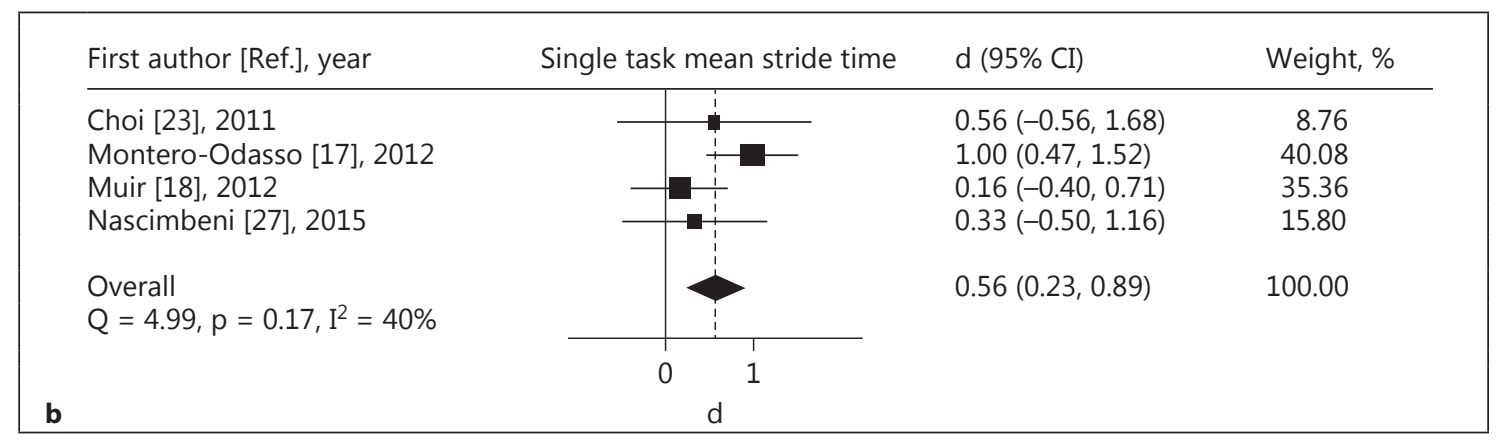

\begin{tabular}{|c|c|c|c|}
\hline First author [Ref.], year & $\begin{array}{l}\text { Counting }(7 ' s) \text { dual task } \\
\text { mean stride time }\end{array}$ & $d(95 \%$ CI) & Weight, \% \\
\hline $\begin{array}{l}\text { Montero-Odasso [17], } 2012 \\
\text { Muir [18], } 2012\end{array}$ & & $\begin{array}{l}0.79(0.28,1.30) \\
1.08(0.48,1.67)\end{array}$ & $\begin{array}{l}57.43 \\
42.57\end{array}$ \\
\hline $\begin{array}{l}\text { Overall } \\
Q=0.50, p=0.48, I^{2}=0 \%\end{array}$ & & $0.91(0.53,1.30)$ & 100.00 \\
\hline & $\begin{array}{l}1 \\
d\end{array}$ & & \\
\hline
\end{tabular}

\begin{tabular}{|lcrr|}
\hline First author [Ref.], year & $\begin{array}{c}\text { Animal naming dual task } \\
\text { mean stride time }\end{array}$ & $\mathrm{d}(95 \% \mathrm{CI})$ & Weight, \% \\
\hline Montero-Odasso [17], 2012 & $0.79(0.27,1.30)$ & 56.58 \\
Muir [18], 2012 & $0.92(0.33,1.50)$ & 43.42 \\
Overall & & $0.84(0.46,1.23)$ & 100.00 \\
$\mathrm{Q}=0.11, \mathrm{p}=0.74, \mathrm{I}^{2}=0 \%$ & & & \\
\hline
\end{tabular}

Fig. 4. Forest plot illustrating the effect of MCI on mean stride length during single task (a), and mean stride time during single task (b), backwards counting by 7's dual task (c) and animal naming dual task (d), compared to cognitively healthy controls. The dotted vertical line corresponds to the overall effect size.

\section{Parameters}

A summary of the 7 studies that included balance assessment for an MCI group is presented in table 4. Twenty-one unique parameters were identified in the included papers, with quantitative balance data presented in 5 of the 7 papers [13, 32-35].

\section{Effect of MCI on Anterior-Posterior Static Balance Parameters}

Sway Variables

For the eyes-open condition, anterior-posterior (AP) mean sway position, measured as the distance from the starting point, was found to be insignificant in significant 


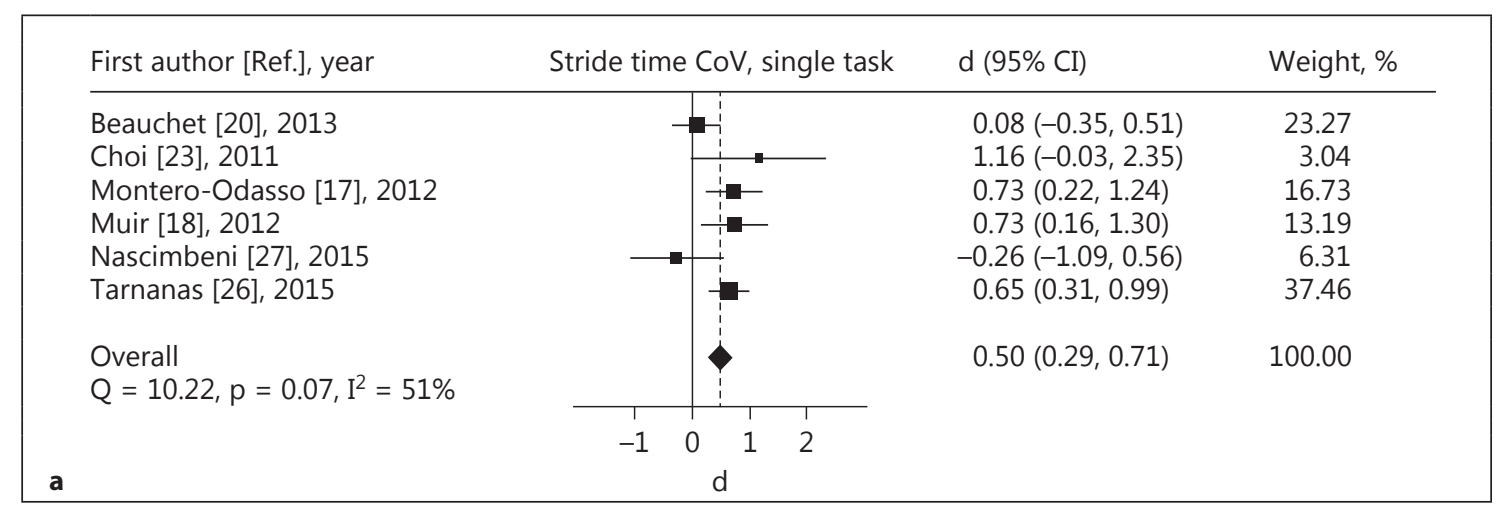

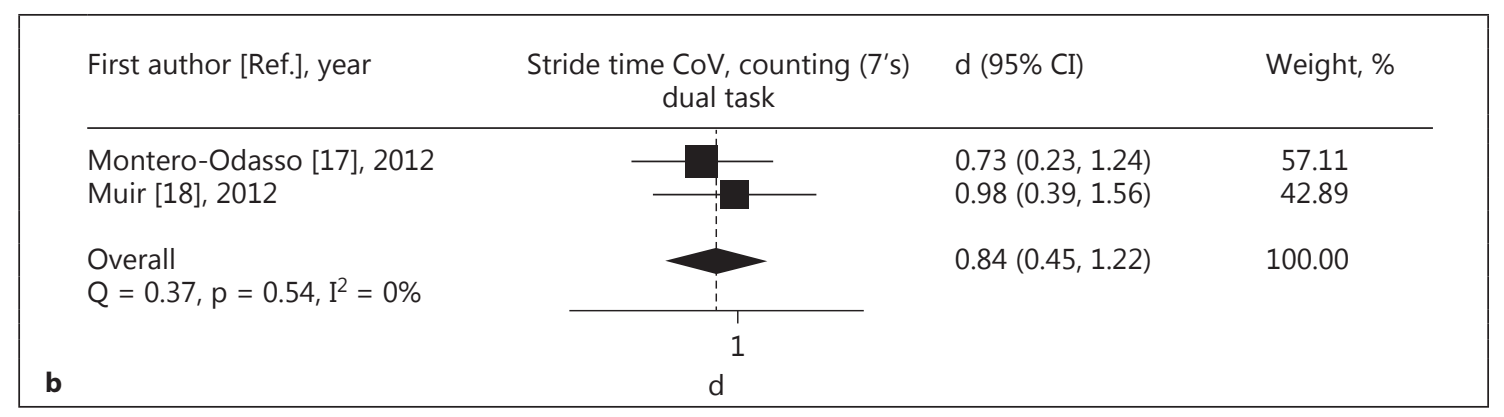

\begin{tabular}{|c|c|c|c|}
\hline First author [Ref.], year & $\begin{array}{c}\text { Stride time CoV, counting (1's) } \\
\text { dual task }\end{array}$ & $d(95 \%$ CI) & Weight, \% \\
\hline $\begin{array}{l}\text { Muir [18], } 2012 \\
\text { Nascimbeni [27], } 2015 \\
\text { Tarnanas [26], } 2015\end{array}$ & $\underset{\frac{1}{1}}{\frac{1}{-1}}$ & $\begin{array}{l}0.97(0.38,1.56) \\
0.42(-0.42,1.25) \\
0.89(0.55,1.24)\end{array}$ & $\begin{array}{l}23.01 \\
11.38 \\
65.60\end{array}$ \\
\hline $\begin{array}{l}\text { Overall } \\
\mathrm{Q}=1.26, \mathrm{p}=0.53, \mathrm{I}^{2}=0 \%\end{array}$ & & $0.86(0.58,1.14)$ & 100.00 \\
\hline & $d^{1}$ & & \\
\hline
\end{tabular}

\begin{tabular}{|c|c|c|c|}
\hline First author [Ref.], year & $\begin{array}{c}\text { Stride time CoV, animal naming } \\
\text { dual task }\end{array}$ & $d(95 \%$ CI) & Weight, \% \\
\hline $\begin{array}{l}\text { Montero-Odassso [17], } 2012 \\
\text { Muir [18], 2012 } \\
\text { Tarnanas [26], } 2015\end{array}$ & 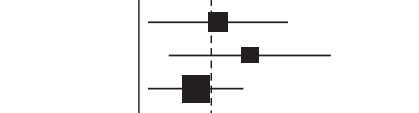 & $\begin{array}{l}0.56(0.05,1.06) \\
0.78(0.21,1.36) \\
0.40(0.07,0.73)\end{array}$ & $\begin{array}{l}24.92 \\
18.95 \\
56.13\end{array}$ \\
\hline $\begin{array}{l}\text { Overall } \\
Q=1.32, p=0.52, I^{2}=0 \%\end{array}$ & & $0.51(0.26,0.76)$ & 100.00 \\
\hline & $\begin{array}{lll}0 & & 1\end{array}$ & & \\
\hline
\end{tabular}

Fig. 5. Forest plot illustrating the effect of MCI on the CoV during single task (a), counting backwards by 7's dual task (b), counting backwards by 1's dual task (c) and animal naming dual task (d) when compared to cognitively healthy controls. The dotted vertical line corresponds to the overall effect size. 
Table 4. Summary of included studies involving balance and MCI

\begin{tabular}{|c|c|c|c|c|}
\hline $\begin{array}{l}\text { First author [Ref.], } \\
\text { year }\end{array}$ & Study characteristics ${ }^{1}$ & Instrumented assessment & Instrument & $\begin{array}{l}\text { Significant balance results } \\
\text { in MCI group }{ }^{2}\end{array}$ \\
\hline $\begin{array}{l}\text { Deschamps [29], } \\
2013\end{array}$ & $\begin{array}{l}\text { Criteria: (a) MMSE, (b) FAB, } \\
\text { (c) ADAS-cog, (d) TMT parts } \\
\text { A/B, (f) Free and Cued Selective } \\
\text { Reminding Test, (g) IADL, } \\
\text { (h) MRI } \\
\text { CHI: } \mathrm{n}=150,76.4 \text { years, } 30 \% \\
\text { MCI: } \mathrm{n}=64,77.5 \text { years, } 39 \% \\
\text { MMAD: } \mathrm{n}=61,78.4 \text { years, } 62 \%\end{array}$ & $\begin{array}{l}\text { Stance with EO } \\
\text { Stance with EC }\end{array}$ & Force platform & $\begin{array}{l}\uparrow \mathrm{COP} \text { mean velocity, EO } \\
\text { and EC; } \uparrow \mathrm{COP} \text { ML mean } \\
\text { velocity, EO; } \\
\uparrow \mathrm{COP} \text { AP average absolute } \\
\text { mean velocity, EO and EC }\end{array}$ \\
\hline $\begin{array}{l}\text { Gillain [11], } \\
2007\end{array}$ & $\begin{array}{l}\text { Criteria: Petersen et al. }[5] \\
\text { CHI: } \mathrm{n}=14,73.5 \text { years, } 21 \% \\
\text { MCI: } \mathrm{n}=14,72.9 \text { years, } 21 \% \\
\text { DEM: } \mathrm{n}=6,73.7 \text { years, } 9 \%\end{array}$ & $\begin{array}{l}\text { Single-leg balance test } \\
\text { Single-leg balance test with } \\
\text { dual task (countdown from } \\
\text { 50) } \\
\text { Pull test } \\
\text { TUG test } \\
\text { TUG test with dual task } \\
\text { (countdown from 50) }\end{array}$ & $\begin{array}{l}\text { Locometrix }^{\circledR} \text { tri-axial } \\
\text { accelerometers }\end{array}$ & $\begin{array}{l}\text { MCI presents intermediate } \\
\text { values, no significant static } \\
\text { balance differences }\end{array}$ \\
\hline $\begin{array}{l}\text { Mignardot [31], } \\
2014\end{array}$ & $\begin{array}{l}\text { Criteria: Winblad et al. }[16] \\
\text { CHI: } \mathrm{n}=228,72.5 \text { years, } 40.3 \% \\
\text { MCI: } \mathrm{n}=140,74.7 \text { years, } 34.3 \% \\
\text { MMAD: } \mathrm{n}=243,83 \text { years, } 61.7 \%\end{array}$ & $\begin{array}{l}\text { TUG test } \\
\text { Stance with EO } \\
\text { Stance with EC }\end{array}$ & Biorescue force platform & $\begin{array}{l}\uparrow \text { COP AP velocity with } \uparrow \\
\text { cognitive impairment }\end{array}$ \\
\hline \multicolumn{5}{|c|}{$\begin{array}{l}\uparrow=\text { Increased; ADAS-cog = Alzheimer's Disease Assessment Scale-Cognitive; } \mathrm{CDR}=\mathrm{Clin} \\
\text { of pressure; DEM = dementia; EC = eyes closed; } \mathrm{EO}=\text { eyes open; } \mathrm{FAB}=\text { Frontal Assessment } \\
\text { MMAD = mild-to-moderate Alzheimer's disease; } \mathrm{MMSE}=\text { Mini-Mental State Examination; } \mathrm{M} \\
\text { TUG = timed up and go. } \\
{ }^{1} \text { Number, mean age, } \% \text { female. } \\
{ }^{2} \text { Compared to an age-matched cognitively healthy control group, if present in the study. }\end{array}$} \\
\hline
\end{tabular}

in 1 paper [29] but not in the eyes-open condition [30]. Meta-analysis of 2 papers [29, 30] found a small-to-medium effect size for AP mean sway position $(\mathrm{d}=0.49,95 \%$ CI, 0.16-0.82, $\mathrm{p}=0.04$; fig. 6a).

For the eyes-closed condition, the AP mean position was found to significantly increase in the eyes-closed condition in 1 study [30] but not in another [36]. Meta-analysis of 2 papers $[29,30]$ showed a medium but not significant effect of MCI on the mean position in the eyesclosed condition $(\mathrm{d}=0.55,95 \% \mathrm{CI},-0.55$ to $1.65, \mathrm{p}=0.33$; fig. 6b).

\section{Sway Velocity Variable}

For the AP sway velocity, 1 paper [29] found that MCI led to a significant increase in trunk velocity for both mean and average absolute maximum values, while others found that neither the sway speed [37] nor the average absolute maximum velocity [31] were significantly affect- ed by MCI. Meta-analysis of average absolute maximum velocity for 2 papers $[29,31]$ identified a small significant effect of MCI in the eyes-open condition ( $d=0.26,95 \%$ CI, 0.08-0.45, $\mathrm{p}<0.01$; fig. 7a).

For the eyes-closed condition, the AP average absolute maximum velocity was found to significantly increase in the eyes-closed condition in 1 study [29] but not in another [31]. The meta-analysis of average absolute maximum velocity for 2 papers $[29,31]$ identified a small significant effect of MCI in the eyes-closed condition ( $\mathrm{d}=$ $0.23,95 \% \mathrm{CI}, 0.05-0.41, \mathrm{p}=0.01$; fig. $7 \mathrm{~b}$ ).

\section{Effect of MCI on Mediolateral Static Balance \\ Parameters}

Sway Position Variables

For the eyes-open condition, 1 paper [30] reported an insignificant effect on mediolateral (ML) sway position and another [29] reported a significant effect. The meta- 


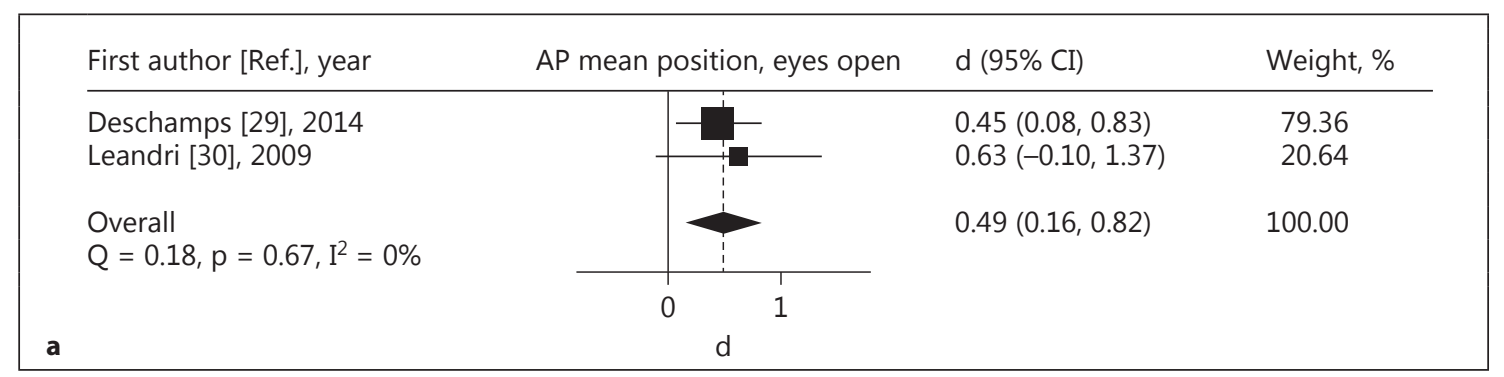

\begin{tabular}{|c|c|c|c|}
\hline First author [Ref.], year & AP mean position, eyes closed & $d(95 \%$ CI $)$ & Weight, \% \\
\hline $\begin{array}{l}\text { Deschamps [29], } 2014 \\
\text { Leandri [30], } 2009\end{array}$ & & $\begin{array}{l}0.04(-0.33,0.41) \\
0.17(0.39,1.95)\end{array}$ & $\begin{array}{l}54.78 \\
45.22\end{array}$ \\
\hline $\begin{array}{l}\text { Overall } \\
Q=6.60, p=0.01, I^{2}=85 \%\end{array}$ & & $0.55(-0.55,1.65)$ & 100.00 \\
\hline & $\begin{array}{lll}0 & 1 & 2 \\
{ }_{d} & \end{array}$ & & \\
\hline
\end{tabular}

\begin{tabular}{|c|c|c|c|c|}
\hline & First author [Ref.], year & ML mean position, eyes open & d $(95 \%$ CI) & Weight, \% \\
\hline & $\begin{array}{l}\text { Deschamps [29], } 2014 \\
\text { Leandri [30], 2009 }\end{array}$ & & $\begin{array}{l}-0.39(-0.76,-0.01) \\
-0.15(-0.87,0.56)\end{array}$ & $\begin{array}{l}78.66 \\
21.34\end{array}$ \\
\hline & $\begin{array}{l}\text { Overall } \\
\mathrm{Q}=0.32, \mathrm{p}=0.57, \mathrm{I}^{2}=0 \%\end{array}$ & & $-0.34(-0.67,-0.01)$ & 100.00 \\
\hline c & & $\mathrm{d}^{0}$ & & \\
\hline
\end{tabular}

\begin{tabular}{|c|c|c|c|}
\hline First author [Ref.], year & ML mean position, eyes closed & $d(95 \%$ CI) & Weight, \% \\
\hline $\begin{array}{l}\text { Deschamps [29], } 2014 \\
\text { Leandri [30], 2009 }\end{array}$ & & $\begin{array}{c}-0.41(-0.78,-0.03) \\
0.42(-0.30,1.15)\end{array}$ & $\begin{array}{l}57.28 \\
45.72\end{array}$ \\
\hline $\begin{array}{l}\text { Overall } \\
\mathrm{Q}=3.98, \mathrm{p}=0.05, \mathrm{I}^{2}=75 \%\end{array}$ & & $-0.05(-0.86,0.75)$ & 100.00 \\
\hline & $\begin{array}{ll}0 & 1 \\
d & \end{array}$ & & \\
\hline
\end{tabular}

Fig. 6. Forest plot illustrating the effect of MCI on AP mean position in the eyes-open (a) and eyes-closed (b) condition, and on ML mean position in the eyes-open (c) and eyes-closed condition (d) compared to cognitively healthy controls. The dotted vertical line corresponds to the overall effect size.

analysis revealed that a small and significant effect was found in the eyes-open condition $(\mathrm{d}=-0.34,95 \% \mathrm{CI}$, -0.67 to $-0.01, \mathrm{p}=0.04$; fig. $6 \mathrm{c}$ ). The meta-analysis of 2 papers $[29,30]$ revealed no significant effect on ML mean position in the eyes-closed condition $(\mathrm{d}=-0.05,95 \% \mathrm{CI}$, -0.86 to $0.75, \mathrm{p}=0.48$; fig. $6 \mathrm{~d}$ ).

\section{Sway Velocity Variables}

One paper [36] found that sway speed increased significantly compared to healthy controls in the eyes-open condition, while 2 papers $[30,37]$ reported a nonsignificant difference. For the eyes-closed condition, 1 paper [36] reported that MCI caused a significant increase in sway speed and position, while another [30] reported a significant difference only with eyes closed that disappeared with post hoc tests. 


\begin{tabular}{|c|c|c|c|}
\hline First author [Ref.], year & AP AAMV, eyes open & $d(95 \%$ CI $)$ & Weight, \% \\
\hline $\begin{array}{l}\text { Deschamps [29], } 2014 \\
\text { Mignardot [31], } 2014\end{array}$ & $\frac{1}{-1}$ & $\begin{array}{l}0.37(0.00,0.74) \\
0.23(0.02,0.44)\end{array}$ & $\begin{array}{l}24.25 \\
75.75\end{array}$ \\
\hline $\begin{array}{l}\text { Overall } \\
Q=0.40, p=0.53, I^{2}=0 \%\end{array}$ & & $0.26(0.08,0.45)$ & 100.00 \\
\hline & $\begin{array}{cl}0 & 0.20 .40 .6 \\
d\end{array}$ & & \\
\hline
\end{tabular}

\begin{tabular}{|c|c|c|c|}
\hline First author [Ref.], year & AP AAMV, eyes closed & $d(95 \%$ CI $)$ & Weight, \% \\
\hline $\begin{array}{l}\text { Deschamps [29], } 2014 \\
\text { Mignardot [31], } 2014\end{array}$ & + & $\begin{array}{l}0.33(-0.04,0.70) \\
0.20(-0.01,0.41)\end{array}$ & $\begin{array}{l}24.28 \\
75.72\end{array}$ \\
\hline $\begin{array}{l}\text { Overall } \\
\mathrm{O}=0.40, \mathrm{p}=0.53, \mathrm{I}^{2}=0 \%\end{array}$ & & $0.23(0.05,0.41)$ & 100.00 \\
\hline & $\begin{array}{cccc}0 & 0.2 & 0.4 & 0.6 \\
& d\end{array}$ & & \\
\hline
\end{tabular}

Fig. 7. Forest plot illustrating the effect of MCI on AP absolute average maximum velocity (AAMV) in the eyesopen (a) and eyes-closed conditions (b) compared to cognitively healthy controls. The dotted vertical line corresponds to the overall effect size.

\section{Qualitative Results}

Increased cognitive impairment was associated with increased velocity standard deviation [29] and absolute average maximum velocity increase [29] in static balance, supporting our findings from the meta-analysis that individuals with MCI have increased postural sway during standing.

\section{Discussion}

Overall, this systematic review and meta-analysis provides sound evidence that MCI adversely affects gait and balance. To our knowledge, this is the first systematic review that provides a comprehensive overview and metaanalysis of studies using objective instrumented assessment of gait and balance. Using this approach, we were able to extract a variety of parameters in both gait and balance in order to identify the most sensitive parameters related to MCI. Our results show that MCI has a substantial impact on specific gait variables. Moreover, we found that static balance is also affected by MCI, indicating that early cognitive changes have a measurable effect on the postural control system and puts patients at increased risk of balance failures and falls.

\section{Changes in Gait Parameters}

Single-Task Conditions

This systematic review demonstrates that gait performance is reduced in people with MCI as reflected by changes in a number of spatiotemporal parameters. When gait is assessed under single-task conditions, gait velocity showed a large effect size for discriminating between MCI and cognitively intact individuals, indicating that this parameter plays a key role in MCI. This result is in line with a number of studies that have identified reduced gait velocity as a predictor for adverse health events including mortality, frailty, or functional dependence $[32,38,39]$. However, slow gait is a nonspecific variable, which is also linked to aging and many aging-related gait disorders. Assessment of gait velocity alone does not provide insight into the specific gait pattern related to MCI, which in turn may limit the sensitivity and specificity of discrimination between people with MCI and cognitively intact.

\section{Dual-Task Conditions}

The use of a dual-task paradigm exposes deficits through the evaluation of activities which simultaneously demand attention resources [40]. One of the main findings of our systematic summary and meta-analysis is that 
dual-task gait assessment increases the sensitivity of gait analysis for discriminating between MCI and healthy groups. Effect sizes were substantially higher for spatiotemporal variables as compared to single task. This information is of high relevance when designing a protocol for diagnosing MCI-specific gait changes and for documenting the impact of specific interventions.

Moreover, we performed a meta-analysis for analyzing the impact of different cognitive tasks used in dualtask protocols. One interesting finding is that the sensitivity of dual-task gait assessment differs depending on the cognitive task used. Arithmetic tasks with a high cognitive demand $(-7)$ have the highest sensitivity, which may have important clinical implications. These findings suggest that a high cognitive load is required in a dualtask protocol for making MCI-specific gait changes emerge. The use of adequate cognitive tasks has been extensively discussed in the literature on cognitively healthy subjects [41, 42] and dementia patients [18, 33, 34, 43]. In dementia, simple cognitive tasks seem to be more appropriate because complex tasks may be too demanding and hamper a reliable dual-task assessment [35]. However, in MCI, it has been less clear which cognitive task is best for high sensitivity of gait analysis. Based on our results, it seems that increasing cognitive demand increases sensitivity. Verbal fluency tasks such as animal naming appear to have a lesser demand than arithmetic tasks because it uses semantic memory as opposed to working memory [44]. In contrast, a low-demand arithmetic task $(-1)$ had very similar results to single-task conditions because it is more rhythmic and may act to cue step patterns [18].

\section{Spatiotemporal Features of Gait}

In our meta-analysis, we identified several gait parameters beyond velocity, which may help to indicate MCIrelated gait changes. The meta-analysis revealed that MCI affects stride time in both single- and dual-task conditions. Although the effect sizes are smaller when compared to gait velocity, once again the largest effect appears in arithmetic dual task. Stride length data was only available for single-task assessments, but also showed that MCI had a significant effect. These two results suggest that the effect of MCI on gait velocity is due to both spatial and temporal modifications in gait.

Variability of stride time provides a measure of gait stability from stride to stride [45]. Calculated effect size from the reviewed studies suggests that increased stride time variability has moderate-to-high power to discriminate between MCI and healthy groups, depending on the

Impact of MCI on Balance and Gait condition (i.e. single task vs. dual task) and thus may serve as an additional parameter for early diagnosis of MCI-related gait deficits. Stride time variability in dual task has been repeatedly reported as a sensitive indicator of cognitive change $[17,46]$.

It has been identified that participant walking strategy changes with distance traveled, resulting in a significant effect on gait variability [47]. The finding of our review supports the influence of walking distance on measuring MCI-related changes in gait variability. For example, in a paper using a 6-meter GAITRite, single task, dual task backwards counting (7's) and animal naming CoV were not significant [18]. In contrast, in a paper using 10-meter GAITRite, all 3 of these values were reported to be significant [26]. These results suggest that a sufficient walking distance is highly relevant in order to measure gait variability as a marker for MCI.

Additionally, we found some evidence that fast-pace walking increases sensitivity for diagnosis MCI-related gait changes. Further, we found that MCI-specific gait changes may particularly emerge during gait initiation. While we could not perform a meta-analysis because only a single study was available, findings may indicate that MCI-related gait changes emerge during more demanding gait situations (i.e., fast walking) and more demanding gait phases (i.e., gait initiation). Similar to dual-task walking (i.e., cognitive stress test), fast walking (i.e. motor stress test) might be helpful in order to identify gait changes in MCI.

\section{Changes in Balance Parameters}

This systematic review shows that MCI has significant effects on static postural balance. A meta-analysis of both AP and ML sway position identified small-to-medium effect sizes that were significant in the eyes-open but not the eyes-closed condition. Although these subtle changes in postural sway may not have a severe impact on ADL, they may indicate a progression toward more severe impairment.

During eyes-open balance testing, visual information is processed for maintaining balance. Research suggests that people with MCI have deficits in processing visual information [48] that results in increased postural sway during balance testing, as discussed previously [49]. Our results support this theory and suggest that MCI-related balance deficits are related impaired central processing of visual information that is critical for balance control.

Limited effects observed during the eyes-closed condition might be related to lack of reliability of static balance 
testing in this specific condition. It was identified in a paper by Helbostad et al. [50] that eyes-closed balance assessments seem to be less reliable than the same assessments in the eyes-open condition.

Another interesting finding was that AP sway speed and mean position was found to have greater changes with MCI than ML in both qualitative and quantitative analysis. In a past study, Franssen et al. [51] identified that persons with MCI had poorer performance in tests of equilibrium and limb coordination. Our results support this, and reveal that AP sway position may be the most sensitive balance parameter for early discrimination of $\mathrm{MCI}$ and $\mathrm{CHI}$. AP sway in static balance is more frequently involved in body stability than ML due to the range of motion available for the body [30]; this natural range could explain the larger effect size of AP mean position as compared to ML mean position in the eyes-open condition.

\section{Implications for Clinical Intervention}

One major strength of this review is that we performed a meta-analysis using only studies which provided a clinically established MCI definition. Our results show that, overall, dual task assessment is the most sensitive tool for gait-based MCI screening. This is an important step forward in developing a clinically validated approach for measuring MCI-related motor deficits, although further studies are required in order to validate the findings of this review.

Information of this review could be useful for promoting specific interventions aiming reverse early motor changes associated with MCI. It has been shown that multicomponent exercise (e.g., aerobic exercise, muscle strength training, gait training) improves gait velocity and stride length in MCI participants [52], Progressive resistance and functional training has been shown to be effective for improving fast walking speed in cognitively impaired individuals. However, there is still room for improvement in current interventions, including specific tailoring to the motor deficits found in this review. For instance, there is limited evidence on intervention effects on stride time variability [9] although this parameter seems to play a critical role in MCI syndrome. New gait training paradigms have shown that gait variability can be influenced in cognitively intact subjects, but studies have not yet been performed in the target population of MCI. It remains to be determined if specific motor learning exercise programs for walking (e.g., overground and treadmill) designed to reinforce rhythmic stepping [53] are effective for reducing gait variability.
Additionally, we found some evidence that MCI-related gait disturbances appear specifically under demanding situations, such as fast walking [20]. This suggests that exercise training in MCI patients should include challenging gait tasks focusing on improvement of gait control in situations with both increased motor (i.e. fast walking) and cognitive (i.e. dual tasking) demand. There is some evidence that gait velocity can be improved in the cognitively impaired under both motor and cognitively challenging conditions [54], using a combination of dualtask training, and progressive strength and functional training $[9,55]$. However, further studies are required in larger populations in order to investigate the effect of this training on important clinical outcomes such as progression of MCI or fall risk.

Importantly, we identified that MCI significantly impacts ML and AP balance control during the eyes-open condition. This opens opportunities for novel intervention paradigms aiming to retrain visual processing of information relevant for postural balance. For instance, it was identified that both MCI patients and age-matched controls use similar compensation strategies for maintaining static balance when provided visual feedback, indicating that compensation systems are intact and may be a target for balance training $[56,57]$.

An interesting study demonstrated that 'non-motor cognitive dual-task training' resulted in motor performance benefits for healthy older adults [58]. This suggests that cognitive training may be an excellent addition to existing training paradigms, particularly for persons with limited mobility.

\section{Limitations}

A lack of uniformity among the study design (e.g., walking distance, variables measured, and instrument) may have affected the validity of analysis for the statistical measurements. The number of parameters included in each meta-analysis varied, depending on the number of studies which reported a specific parameter. This may have biased our findings. For parameters which were more frequently reported (e.g., gait velocity), the metaanalysis results are more precise. Furthermore, funnel plot analysis suggests the presence of a publication which may have affected the validity of our analysis. In performing meta-analyses, our pragmatic approach was to include the maximum number of studies reporting each parameter in order to accurately evaluate the evidence that is currently available.
Bahureksa/Najafi/Saleh/Sabbagh/Coon/ Mohler/Schwenk 
Speed dependency of gait variables was not discussed in this paper since only 1 paper [20] contained data at a fast walking speed. Time-to-boundary measures, or nonlinear measures of postural sway, were not examined in these papers but may provide information on more subtle changes in motor control in the MCI population. We acknowledge that more studies using a standardized instrumented assessment procedure are required to verify the validity of our results

\section{Conclusion and Clinical Implications}

The use of motor performance measures, particularly under cognitively challenging conditions (i.e. dual task), may provide a sensitive, early, and non-invasive means for screening of clinically relevant MCI-specific motor disturbances. Identification of early gait and MCI deficits could provide a critical opportunity for early intervention before gait and balance changes have a major impact on ADLs, fall risk, and overall independence. This review provides sound evidence on which parameters should be used in gait and balance assessment, and provides a basis for future studies aiming to further develop, verify, and refine a standardized clinical motor assessment protocol for people with MCI.

\section{Acknowledgements}

This research was performed in collaboration with the interdisciplinary Consortium on Advanced Motion Performance (iCAMP) at the University of Arizona, Banner Sun Health Research Institute, the Arizona Center on Aging, and Arizona State University. It was funded in part by the Flinn Foundation: Arizona Aging and Cognitive Collaborative grant (award number 1907), the Undergraduate Biology Research Program (HHMI 52006942), and by the National Institute on Aging (award numbers 2R42AG032748 and P30 AG019610). The sponsors had no role in the design or conduct of the study; collection, management, analysis, or interpretation of the data; or preparation, review, or approval of the manuscript. We thank Charles Huang and Tulcy Patel who assisted with the screening process. The authors would like to thank Qianzi Zhang, MSc, a member of the Michael E. DeBakey Department of Surgery Research Core at Baylor College of Medicine, for her assistance in the meta-analysis during the preparation of the manuscript.

\section{Disclosure Statement}

All authors report no conflict of interest or any financial support.

\section{References}

1 Huang C, Mattis P, Julin P: Identifying functional imaging markers of mild cognitive impairment in early Alzheimer's and Parkinson's disease using multivariate analysis. Clin Neurosci Res 2007;6:367-373.

2 Dierckx E, Engelborghs S, De Raedt R, De Deyn PP, Ponjaert-Kristoffersen I: Mild cognitive impairment: what's in a name? Gerontology 2007;53:28-35.

-3 Petersen RC, Roberts RO, Knopman DS, Geda YE, Cha RH, Pankratz VS, Boeve BF, Tangalos EG, Ivnik RJ, Rocca WA: Prevalence of mild cognitive impairment is higher in men. The Mayo Clinic Study of Aging. Neurology 2010;75:889-897.

4 Brookmeyer R, Johnson E, Ziegler-Graham $\mathrm{K}$, Arrighi HM: Forecasting the global burden of Alzheimer's disease. Alzheimers Dement 2007;3:186-191.

5 Petersen RC: Mild cognitive impairment as a diagnostic entity. J Intern Med 2004;256:183194.

6 Dubois B, Albert ML: Amnestic MCI or prodromal Alzheimer's disease? Lancet Neurol 2004;3:246-248.
Portet F, Ousset PJ, Visser PJ, Frisoni GB, Nobili F, Scheltens P, Vellas B, Touchon J: Mild cognitive impairment (MCI) in medical practice: a critical review of the concept and new diagnostic procedure. Report of the MCI Working Group of the European Consortium on Alzheimer's Disease. J Neurol Neurosurg Psychiatry 2006;77:714-718.

8 Burton CL, Strauss E, Bunce D, Hunter MA, Hultsch DF: Functional abilities in older adults with mild cognitive impairment. Gerontology 2009;55:570-581.

-9 Schwenk M, Zieschang T, Englert S, Grewal G, Najafi B, Hauer K: Improvements in gait characteristics after intensive resistance and functional training in people with dementia: a randomised controlled trial. BMC Geriatr 2014; $14: 73$

10 Schaefer S, Schumacher V: The interplay between cognitive and motor functioning in healthy older adults: findings from dual-task studies and suggestions for intervention. Gerontology 2011;57:239-246.
1 Gillain S, Warzee E, Lekeu F, Wojtasik V, Maquet D, Croisier JL, Salmon E, Petermans J: The value of instrumental gait analysis in elderly healthy, MCI or Alzheimer's disease subjects and a comparison with other clinical tests used in single and dual-task conditions. Ann Phys Rehabil Med 2009;52:453-474.

12 Verghese J, Lipton RB, Hall CB, Kuslansky G, Katz MJ, Buschke H: Abnormality of gait as a predictor of non-Alzheimer's dementia. $\mathrm{N}$ Engl J Med 2002;347:1761-1768.

13 Granacher U, Bridenbaugh SA, Muehlbauer T, Wehrle A, Kressig RW: Age-related effects on postural control under multi-task conditions. Gerontology 2011;57:247-255.

-14 Tell GS, Lefkowitz DS, Diehr P, Elster AD: Relationship between balance and abnormalities in cerebral magnetic resonance imaging in older adults. Arch Neurol 1998;55:73-79.

15 Moher D, Liberati A, Tetzlaff J, Altman DG, RPISAM Group: Preferred reporting items for systematic reviews and meta-analyses: the PRISMA statement. PLoS Med 2009; 6:e1000097. 
16 Winblad B, Palmer K, Kivipelto M, Jelic V, Fratiglioni L, Wahlund LO, Nordberg A, Backman L, Albert M, Almkvist O, Arai H, Basun H, Blennow K, de Leon M, DeCarli C, Erkinjuntti T, Giacobini E, Graff C, Hardy J, Jack C, Jorm A, Ritchie K, van Duijn C, Visser P, Petersen RC: Mild cognitive impairment - beyond controversies, towards a consensus: report of the International Working Group on Mild Cognitive Impairment. J Intern Med 2004;256:240-246.

17 Montero-Odasso M, Muir SW, Speechley M: Dual-task complexity affects gait in people with mild cognitive impairment: the interplay between gait variability, dual tasking, and risk of falls. Arch Phys Med Rehabil 2012;93:293299.

18 Muir SW, Speechley M, Wells J, Borrie M, Gopaul K, Montero-Odasso M: Gait assessment in mild cognitive impairment and Alzheimer's disease: the effect of dual-task challenges across the cognitive spectrum. Gait Posture 2012;35:96-100.

19 Cohen J: Statistical Power Analysis for the Behavior Sciences, ed 2. Hillsdale, Lawrence Erlbaum Associates, 1988.

20 Beauchet O, Allali G, Launay C, Herrmann FR, Annweiler C: Gait variability at fast-pace walking speed: a biomarker of mild cognitive impairment? J Nutr Health Aging 2013;17: 235-239.

21 Beauchet O, Allali G, Thiery S, Gautier J, Fantino B, Annweiler C: Association between high variability of gait speed and mild cognitive impairment: a cross-sectional pilot study. J Am Geriatr Soc 2011;59:1973-1974.

-22 Boripuntakul S, Lord SR, Brodie MA, Smith ST, Methapatara P, Wongpakaran N, Sungkarat S: Spatial variability during gait initiation while dual tasking is increased in individuals with mild cognitive impairment. J Nutr Health Aging 2014; 18:307-312.

23 Choi JS, Oh HS, Kang DW, Mun KR, Choi MH, Lee SJ, Yang JW, Chung SC, Mun SW, Tack GR: Comparison of gait and cognitive function among the elderly with Alzheimer's disease, mild cognitive impairment and healthy. Int J Precis Eng Manuf 2011;12:169173.

24 Verghese J, Robbins M, Holtzer R, Zimmerman M, Wang C, Xue X, Lipton RB: Gait dysfunction in mild cognitive impairment syndromes. J Am Geriatr Soc 2008;56:12441251.

-25 Montero-Odasso M, Oteng-Amoako A, Speechley M, Gopaul K, Beauchet O, Annweiler C, Muir-Hunter SW: The motor signature of mild cognitive impairment: results from the gait and brain study. J Gerontol A Biol Sci Med Sci 2014;69:1415-1421.

-26 Tarnanas I, Papagiannopoulos S, Kazis D, Wiederhold M, Widerhold B, Tsolaki M: Reliability of a novel serious game using dual-task gait profiles to early characterize aMCI. Front Aging Neurosci 2015;7:50.
Nascimbeni A, Caruso S, Salatino A, Carenza M, Rigano M, Raviolo A, Ricci R: Dual taskrelated gait changes in patients with mild cognitive impairment. Funct Neurol 2015;30:5965.

28 Maquet D, Lekeu F, Warzee E, Gillain S, Wojtasik V, Salmon E, Petermans J, Croisier JL: Gait analysis in elderly adult patients with mild cognitive impairment and patients with mild Alzheimer's disease: simple versus dual task: a preliminary report. Clin Physiol Funct Imaging 2010;30:51-56.

29 Deschamps T, Beauchet O, Annweiler C, Cornu C, Mignardot JB: Postural control and cognitive decline in older adults: position versus velocity implicit motor strategy. Gait Posture 2014;39:628-630.

30 Leandri M, Cammisuli S, Cammarata S, Baratto L, Campbell J, Simonini M, Tabaton M: Balance features in Alzheimer's disease and amnestic mild cognitive impairment. J Alzheimers Dis 2009;16:113-120.

-31 Mignardot JB, Beauchet O, Annweiler C, Cornu C, Deschamps T: Postural sway, falls, and cognitive status: a cross-sectional study among older adults. J Alzheimers Dis 2014;41: 431-439.

32 Cesari M, Kritchevsky SB, Penninx BW, Nicklas BJ, Simonsick EM, Newman AB, Tylavsky FA, Brach JS, Satterfield S, Bauer DC, Visser M, Rubin SM, Harris TB, Pahor M: Prognostic value of usual gait speed in wellfunctioning older people - results from the Health, Aging and Body Composition Study. J Am Geriatr Soc 2005;53:1675-1680.

33 Barberger-Gateau P, Fabrigoule C: Disability and cognitive impairment in the elderly. Disabil Rehabil 1997;19:175-193.

34 Redfern MS, Muller ML, Jennings JR, Furman JM: Attentional dynamics in postural control during perturbations in young and older adults. J Gerontol A Biol Sci Med Sci 2002; 57:B298-B303.

35 Holtzer R, Verghese J, Xue X, Lipton RB: Cognitive processes related to gait velocity: results from the Einstein Aging Study. Neuropsychology 2006;20:215-223.

36 Shin BM, Han SJ, Jung JH, Kim JE, Fregni F: Effect of mild cognitive impairment on balance. J Neurol Sci 2011;305:121-125.

37 Jeon SY, Han SJ, Jeong JH, Fregni F: Effect of exercise on balance in persons with mild cognitive impairment. Neurorehabilitation 2014; 35:271-278.

38 Schwenk M, Howe C, Saleh A, Mohler J, Grewal G, Armstrong D, Najafi B: Frailty and technology: a systematic review of gait analysis in those with frailty. Gerontology 2014;60: 79-89.

39 Gill TM, Williams CS, Tinetti ME: Assessing risk for the onset of functional dependence among older adults: the role of physical performance. J Am Geriatr Soc 1995;43:603-609.

40 Beauchet O, Berrut G: Gait and dual-task: definition, interest, and perspectives in the elderly. Psychol Neuropsychiatr Vieil 2006;4:215225.
41 Beauchet O, Dubost V, Aminian K, Gonthier R, Kressig RW: Dual-task-related gait changes in the elderly: does the type of cognitive task matter? J Motor Behav 2005;37:259-264.

42 Melzer I, Oddsson LI: The effect of a cognitive task on voluntary step execution in healthy elderly and young individuals. J Am Geriatr Soc 2004;52:1255-1262.

43 Camicioli R, Howieson D, Lehman S, Kaye J: Talking while walking: the effect of a dual task in aging and Alzheimer's disease. Neurology 1997;48:955-958.

44 Redfern MS, Muller ML, Jennings JR, Furman JM: Attentional dynamics in postural control during perturbations in young and older adults. J Gerontol A Biol Sci Med Sci 2002; 57:B298-B303.

45 Holtzer R, Verghese J, Xue X, Lipton RB: Cognitive processes related to gait velocity: results from the Einstein Aging Study. Neuropsychology 2006;20:215-223.

-46 Beauchet O, Dubost V, Gonthier R, Kressig RW: Dual-task-related gait changes in transitionally frail older adults: the type of the walking-associated cognitive task matters. Gerontology 2005;51:48-52.

-47 Beauchet O, Annweiler C, Lecordroch Y, Allali G, Dubost V, Herrmann FR, Kressig RW: Walking speed-related changes in stride time variability: effects of decreased speed. J Neuroeng Rehabil 2009;6:32.

48 Lamoth CJ, van Deudekom FJ, van Campen JP, Appels BA, de Vries OJ, Pijnappels M: Gait stability and variability measures show effects of impaired cognition and dual tasking in frail people. J Neuroeng Rehabil 2011;8:2-2.

-49 Najafi B, Helbostad JL, Moe-Nilssen R, Zijlstra W, Aminian K: Does walking strategy in older people change as a function of walking distance? Gait Posture 2009;29:261-266.

50 Perrochon A, Kemoun G, Dugue B, Berthoz A: Cognitive impairment assessment through visuospatial memory can be performed with a modified Walking Corsi Test using the 'magic carpet'. Dement Geriatr Cogn Dis Extra 2014;4:1-13

51 Redfern MS, Jennings JR, Mendelson D, Nebes RD: Perceptual inhibition is associated with sensory integration in standing postural control among older adults. J Geront B Psychol Sci Soc Sc 2009;64:569-576.

52 Helbostad JL, Askim T, Moe-Nilssen R: Short-term repeatability of body sway during quiet standing in people with hemiparesis and in frail older adults. Arch Phys Med Rehabil 2004;85:993-999.

53 Franssen EH, Souren LE, Torossian CL, Reisberg B: Equilibrium and limb coordination in mild cognitive impairment and mild Alzheimer's disease. J Am Geriatr Soc 1999;47: 463-469. 
54 Doi T, Makizako H, Shimada H, Yoshida D, Tsutsumimoto K, Sawa R, Misu S, Suzuki T: Effects of multicomponent exercise on spatial-temporal gait parameters among the elderly with amnestic mild cognitive impairment (aMCI): preliminary results from a randomized controlled trial (RCT). Arch Gerontol Geriatr 2013;56:104-108.

55 Brach JS, Van Swearingen JM, Perera S, Wert DM, Studenski S: Motor learning versus standard walking exercise in older adults with subclinical gait dysfunction: a randomized clinical trial. J Am Geriatr Soc 2013;61:18791886.
6 Schwenk M, Zieschang T, Oster P, Hauer K: Dual-task performances can be improved in patients with dementia: a randomized controlled trial. Neurology 2010;74:1961-1968.

57 Silsupadol P, Lugade V, Shumway-Cook A, van Donkelaar P, Chou LS, Mayr U, Woollacott MH: Training-related changes in dualtask walking performance of elderly persons with balance impairment: a double-blind, randomized controlled trial. Gait Posture 2009;29:634-639.
58 Grewal GS, Schwenk M, Lee-Eng J, Parvaneh S, Bharara M, Menzies RA, Talal TK, Armstrong DG, Najafi B: Sensor-based interactive balance training with visual joint movement feedback for improving postural stability in diabetics with peripheral neuropathy: a randomized controlled trial. Gerontology 2015; 61:567-574.

59 Schwenk M, Grewal GS, Honarvar B, Schwenk S, Mohler J, Khalsa DS, Najafi B: Interactive balance training integrating sensor-based visual feedback of movement performance: a pilot study in older adults. J Neuroeng Rehabil 2014;11:164. 